\title{
THE ASYMPTOTIC DISTRIBUTION OF THE SUM OF A RANDOM NUMBER OF RANDOM VARIABLES
}

\author{
HERBERT ROBBINS
}

1. Introduction. If a random variable (r. v.) $Y$ is the sum of a large but constant number $N$ of independent components

$$
Y=X_{1}+\cdots+X_{N},
$$

then under appropriate conditions on the $X_{j}$ it follows from the central limit theorem that the distribution of $Y$ will be nearly normal. In many cases of practical importance, however, the number $N$ is itself a r. v., and when this is so the situation is more complex.

We shall consider the case in which the $X_{j}(j=1,2, \cdots)$ are independent r. v.'s with the same distribution function (d. f.) $F(x)$ $=P\left[X_{j} \leqq x\right]$, and in which the non-negative integer-valued r. v. $N$ is independent of the $X_{j}$. The d. f. of $N$ we shall assume to depend on a parameter $\lambda$, so that the d. f. of $Y$ is a function of $\lambda$ which may have an asymptotic expression as $\lambda \rightarrow \infty$. In the degenerate case in which for any integer $\lambda, N$ is certain to have the value $\lambda$, the problem reduces to the ordinary central limit problem for equi-distributed components.

In the general case the d. f. of $N$ for any $\lambda$ is determined by the values $\omega_{k}=P[N=k](k=0,1, \cdots)$, where the $\omega_{k}$ are functions of $\lambda$ such that for all $\lambda$,

$$
\omega_{k} \geqq 0, \quad \sum_{0}^{\infty} \omega_{k}=1 .
$$

We shall use Greek letters to denote functions of the parameter $\lambda$; in particular we define

$$
\begin{aligned}
\alpha & =E(N)=\sum_{0}^{\infty} \omega_{k} \cdot k \\
\beta^{2} & \left.=E\left(N^{2}\right)=\sum_{0}^{\infty} \omega_{k} \cdot k^{2} \quad \text { (assumed finite for all } \lambda\right), \\
\gamma^{2} & =\operatorname{Var}(N)=\sum_{0}^{\infty} \omega_{k} \cdot(k-\alpha)^{2}=\beta^{2}-\alpha^{2}, \\
\theta(t) & =E\left(e^{i(N-\alpha) t / \gamma}\right)=\sum_{0}^{\infty} \omega_{k} \cdot e^{i(k-\alpha) t / \gamma},
\end{aligned}
$$

Received by the editors January 12, 1948. 
the last being the characteristic function (c. f.) of the normalized r. v.

$$
M=(N-\alpha) / \gamma .
$$

We shall use Latin letters to denote quantities independent of $\lambda$; in particular we define

$$
\begin{aligned}
a & =E\left(X_{j}\right)=\int x d F(x), \\
b^{2} & =E\left(X_{j}^{2}\right)=\int x^{2} d F(x), \\
c^{2} & =\operatorname{Var}\left(X_{j}\right)=\int(x-a)^{2} d F(x)=b^{2}-a^{2}\left(0<c^{2}<\infty\right), \\
f(t) & =E\left(e^{i X_{j} t}\right)=\int e^{i x t} d F(x) .
\end{aligned}
$$

We then have for the r. v. (1),

$$
\begin{aligned}
E(Y) & =\sum_{0}^{\infty} \omega_{k} \cdot E\left(X_{1}+\cdots+X_{k}\right)=\sum_{0}^{\infty} \omega_{k} \cdot k a=\alpha a, \\
E\left(Y^{2}\right) & =\sum_{0}^{\infty} \omega_{k} \cdot E\left(X_{1}+\cdots+X_{k}\right)^{2} \\
& =\sum_{0}^{\infty} \omega_{k}\left\{k b^{2}+k(k-1) a^{2}\right\}=\alpha c^{2}+\beta^{2} a^{2}, \\
\sigma^{2} & =\operatorname{Var}(Y)=\alpha c^{2}+\gamma^{2} a^{2} .
\end{aligned}
$$

We shall be concerned with the normalized $r$. v.

$$
Z=\frac{Y-E(Y)}{(\operatorname{Var}(Y))^{1 / 2}}=\frac{\left(X_{1}+\cdots+X_{N}\right)-\alpha a}{\sigma},
$$

whose c. f. is

$$
\begin{aligned}
\phi(t)=E\left(e^{i Z t}\right) & =\sum_{0}^{\infty} \omega_{k} \cdot E\left(e^{i\left[\left(X_{1}+\cdots+X_{k}\right)-\alpha a / \sigma\right] t}\right), \\
& =\sum_{0}^{\infty} \omega_{k} \cdot e^{-i \alpha a t / \sigma} \cdot f^{k}\left(\frac{t}{\sigma}\right) .
\end{aligned}
$$

By definition, $Z$ has the limiting d. f. $H(x)$ if whenever $x$ is a continuity point of the d. f. $H(x), \lim _{\lambda \rightarrow \infty} P[Z \leqq x]=H(x)$, or, equivalently, setting 


$$
h(t)=\int e^{i x t} d H(x)
$$

if for every $t$,

$$
\lim _{\lambda \rightarrow \infty} \phi(t)=h(t)
$$

In particular, if (8) holds for $h(t)=e^{-t^{2} / 2}$, then for every $x$,

$$
\lim _{\lambda \rightarrow \infty} P[Z \leqq x]=\frac{1}{(2 \pi)^{1 / 2}} \int_{-\infty}^{x} e^{-u^{2} / 2} d u \equiv H_{0}(x),
$$

and $Y$ is said to be asymptotically normal $(\alpha a, \sigma)$.

\section{Some general results.}

THEOREM 1. Let

$$
\delta=\frac{\gamma a}{\sigma}=\left(\frac{\gamma^{2} a^{2}}{\alpha c^{2}+\gamma^{2} a^{2}}\right)^{1 / 2} \quad(0 \leqq \delta \leqq 1)
$$

If, as $\lambda \rightarrow \infty$,

$$
\sigma^{2} \rightarrow \infty, \quad \gamma=o\left(\sigma^{2}\right),
$$

then

$$
\phi(t)=\theta(\delta t) \cdot e^{-t^{2}\left(1-\delta^{2}\right) / 2}+o(1) .
$$

Proof. Since from (10),

$$
E\left(\frac{N-\alpha}{\sigma^{2}}\right)=0, \quad E\left(\frac{N-\alpha}{\sigma^{2}}\right)^{2}=\frac{\gamma^{2}}{\sigma^{4}} \rightarrow 0,
$$

it follows that $(N-\alpha) / \sigma^{2} \rightarrow 0$ in probability as $\lambda \rightarrow \infty$. Hence for any $d>0$,

$$
2 \cdot P\left[\left|\frac{N-\alpha}{\sigma^{2}}\right|>d\right]=o(1) \quad \text { as } \lambda \rightarrow \infty .
$$

We now write (7) in the form

$$
\phi(t)=\sum_{0}^{\infty} \omega_{k} \cdot e^{i(k-\alpha) a t / \sigma} \cdot\left\{e^{-i a t / \sigma} f\left(\frac{t}{\sigma}\right)\right\}^{k}
$$

and define

$$
\phi_{1}(t)=\left\{\sum_{0}^{\infty} \omega_{k} \cdot e^{i(k-\alpha) a t / \sigma}\right\} \cdot\left\{e^{-i a t / \sigma} f\left(\frac{t}{\sigma}\right)\right\}^{\alpha}
$$


1154

HERBERT ROBBINS

[December

then

$$
\begin{aligned}
\phi(t)-\phi_{1}(t)= & \sum_{0}^{\infty} \omega_{k} \cdot e^{i(k-\alpha) a t / \sigma}\left[\left\{e^{-i a t / \sigma} f\left(\frac{t}{\sigma}\right)\right\}^{k}\right. \\
& \left.-\left\{e^{-i a t / \sigma} f\left(\frac{t}{\sigma}\right)\right\}^{\alpha}\right]
\end{aligned}
$$

whence

$$
\begin{aligned}
\left|\phi(t)-\phi_{1}(t)\right| & \leqq \sum_{0}^{\infty} \omega_{k}\left|\left\{e^{-i a t / \sigma} f\left(\frac{t}{\sigma}\right)\right\}^{k}-\left\{e^{-i a t / \sigma} f\left(\frac{t}{\sigma}\right)\right\}^{\alpha}\right| \\
& =\sum_{\left|(k-\alpha) / \sigma^{2}\right|>d}+\sum_{\left|(k-\alpha) / \sigma^{2}\right| \leqq d} \\
& \leqq 2 \sum_{\left|(k-\alpha) / \sigma^{2}\right|>d} \omega_{k}+\left|e^{-i a t / \sigma} f\left(\frac{t}{\sigma}\right)\right|^{\alpha} \\
& \leqq 2 P\left[\left|\frac{N-\alpha}{\sigma^{2}}\right|>d\right] \\
& +\sum_{|r| \leqq d} \omega_{k}\left|\left\{e^{-i a t / \sigma} f\left(\frac{t}{\sigma}\right)\right\}^{k-\alpha}-1\right|
\end{aligned}
$$

From (4) we have as $t \rightarrow 0$,

$$
f(t)=1+i a t-\frac{b^{2} t^{2}}{2}+o\left(t^{2}\right) ;
$$

hence as $\sigma^{2} \rightarrow \infty$

$$
\begin{aligned}
e^{-i a t / \sigma} f\left(\frac{t}{\sigma}\right)= & \left\{1-\frac{i a t}{\sigma}-\frac{a^{2} t^{2}}{2 \sigma^{2}}+o\left(\frac{1}{\sigma^{2}}\right)\right\} \\
& \cdot\left\{1+\frac{i a t}{\sigma}-\frac{b^{2} t^{2}}{2 \sigma^{2}}+o\left(\frac{1}{\sigma^{2}}\right)\right\} \\
= & 1-\frac{c^{2} t^{2}}{2 \sigma^{2}}+o\left(\frac{1}{\sigma^{2}}\right) \\
\left\{e^{-i a t / \sigma} f\left(\frac{t}{\sigma}\right)\right\}^{\sigma^{2}}= & \left\{1-\frac{c^{2} t^{2}}{2 \sigma^{2}}+o\left(\frac{1}{\sigma^{2}}\right)\right\}^{\sigma^{2}}=e^{-c^{2} t^{2} / 2}+o(1) \\
= & \left\{e^{-t^{2} / 2}+o(1)\right\} c^{2} .
\end{aligned}
$$


Thus

$$
\begin{aligned}
\left\{e^{-i a t / \sigma} f\left(\frac{t}{\sigma}\right)\right\}^{\alpha} & =\left\{e^{-i a t / \sigma} f\left(\frac{t}{\sigma}\right)\right\}^{\sigma^{2} \alpha / \sigma} \\
& =\left\{e^{-t^{2} / 2}+o(1)\right\} c^{2} \alpha / \sigma^{2}
\end{aligned}
$$

and

$$
\left\{e^{-i a t / \sigma} f\left(\frac{t}{\sigma}\right)\right\}^{\sigma r}=\left\{e^{-t^{2} / 2}+o(1)\right\} c^{2} r
$$

Now fix $t$ and $\epsilon>0$. Choose $d>0$, until now arbitrary, so that

$$
\left|z-e^{-t^{2} / 2}\right|<d, \quad|r|<d
$$

imply that

$$
\left|z^{c^{2} r}-1\right|<\frac{\epsilon}{2}
$$

Then choose $\lambda_{1}$ so that $\lambda>\lambda_{1}$ implies that

$$
2 P\left[\left|\frac{N-\alpha}{\sigma^{2}}\right|>d\right]<\frac{\epsilon}{2}
$$

and that the $o(1)$ in (17) satisfies the inequality

$$
|o(1)|<d \text {. }
$$

Then it follows from (15) that for $\lambda>\lambda_{1}$,

$$
\left|\phi(t)-\phi_{1}(t)\right|<\frac{\epsilon}{2}+\frac{\epsilon}{2}=\epsilon ;
$$

since $\epsilon$ was arbitrary we conclude that

$$
\phi(t)=\phi_{1}(t)+o(1) .
$$

We can write $\phi_{1}(t)$ in the form

$$
\begin{aligned}
\phi_{1}(t) & =\left\{\sum_{0}^{\infty} \omega_{k} \cdot e^{i(k-\alpha) a t / \sigma}\right\} \cdot\left\{e^{-i a t / \sigma} f\left(\frac{t}{\sigma}\right)\right\}^{\alpha} \\
& =\theta\left\{\frac{\gamma a t}{\sigma}\right\} \cdot\left\{e^{-t^{2} / 2}+o(1)\right\}^{\alpha c^{2} / \sigma^{2}}=\theta(\delta t) \cdot\left\{e^{-t^{2} / 2}+o(1)\right\}^{1-\delta^{2}} \\
& =\theta(\delta t) \cdot\left\{e^{-t^{2} / 2}(1+o(1))\right\}^{1-\delta^{2}} \\
& =\theta(\delta t) \cdot e^{-t^{2}\left(1-\delta^{2}\right) / 2}+o(1),
\end{aligned}
$$

which, with (22), completes the proof of the theorem. 
CoRollary 1. If (10) holds, and if as $\lambda \rightarrow \infty$,

$$
a^{2} \gamma^{2}=o(\alpha)
$$

then

$$
\lim _{\lambda \rightarrow \infty} \phi(t)=e^{-t^{2} / 2}
$$

so that $Z$ has the limiting d. f. $H_{0}(x)$ and $Y$ is asymptotically normal $(\alpha a, \sigma)$.

Proof. From (24) it follows that as $\lambda \rightarrow \infty, \delta \rightarrow 0$. Moreover, considering the r. $v$.

$$
M_{1}=\frac{(N-\alpha) \delta}{\gamma}
$$

we have $E\left(M_{1}\right)=0, E\left(M_{1}^{2}\right)=\delta^{2} \rightarrow 0$, so that $M_{1} \rightarrow 0$ in probability. It follows that

$$
E\left(e^{i M_{1} t}\right)=\sum_{0}^{\infty} \omega_{k} \cdot e^{i(k-\alpha) \delta t / \gamma}=\theta(\delta t) \rightarrow 1,
$$

while

$$
e^{-t^{2}\left(1-\delta^{2}\right) / 2} \rightarrow e^{-t^{2} / 2},
$$

so that (25) follows from (11).

Until now we have not assumed that the normalized r. v. $M$ defined by (3) has a limiting d. f. $G(x)$ as $\lambda \rightarrow \infty$.

CoROLlary 2. If (10) holds, and if $N$ is asymptotically normal $(\alpha, \gamma)$, then $Z$ has the limiting d. f. $H_{0}(x)$ and $Y$ is asymptotically normal $(\alpha a, \sigma)$.

Proof. In this case we have

$$
\lim _{\lambda \rightarrow \infty} \theta(\tau)=e^{-\tau^{2} / 2},
$$

and the convergence is uniform in the interval $0 \leqq \tau \leqq t$. Since $0 \leqq \delta \leqq 1$ it follows that as $\lambda \rightarrow \infty$,

$$
\theta(\delta t)=e^{-(\delta t)^{2} / 2}+o(1),
$$

and therefore from (11),

$$
\phi(t)=\left\{e^{-\delta^{2} t^{2} / 2}+o(1)\right\} \cdot\left\{e^{-\left(1-\delta^{2}\right) t^{2} / 2}\right\}+o(1)=e^{-t^{2} / 2}+o(1) .
$$

The assumption (10) is actually superfluous in this case as we shall see later (Corollary 4). 
Let us now consider the case in which $M$ has a non-normal limiting d. $f$.

COROLlARy 3. If (10) holds, and if $M$ has a non-normal limiting d. f. $G(x)$, so that

$$
\lim _{\lambda \rightarrow \infty} \theta(t)=g(t)=\int e^{i x t} d G(x) \not \equiv e^{-t^{2} / 2}
$$

and if

$$
\lim _{\lambda \rightarrow \infty} \frac{c^{2} \alpha}{a^{2} \gamma^{2}}=s
$$

exists, $0 \leqq s<\infty$, then

$$
\lim _{\lambda \rightarrow \infty} \phi(t)=g\left(\frac{t}{(1+s)^{1 / 2}}\right) \cdot e^{-\left(t(s /(1+s))^{1 / 2}\right)^{2} / 2} \not \neq e^{-t^{2} / 2},
$$

so that $Z$ has the non-normal limiting $d . f$.

$$
H(x)=G\left(t(1+s)^{1 / 2}\right)_{*} H_{0}\left(x\left(\frac{1+s}{s}\right)^{1 / 2}\right)
$$

where * denotes the operation of convolution.

Proof. In this case as $\lambda \rightarrow \infty, \delta \rightarrow(1+s)^{-1 / 2}$, whence (31) follows as before.

If $s=0$ (that is, if $\alpha=o\left(a^{2} \gamma^{2}\right)$ ) then $\lim _{\lambda \rightarrow \infty} \phi(t)=\lim _{\lambda \rightarrow \infty} \theta(t)=g(t)$, so that $Y$ has the same asymptotic distribution as $N$. If $0<s<\infty$ then the limiting d. f. of $Z$ is the convolution of a normal with a nonnormal d. f. If $s=\infty$ we refer to Corollary 1 .

LEMma 1. If $M$ has a limiting d. $f . G(x)$ such that $G(x)>0$ for every finite $x$, then (10) holds.

Proof. First we shall show that $\gamma=o(\alpha)$ as $\lambda \rightarrow \infty$. Suppose not. Then there exists a constant $B>0$ such that for any $\lambda_{1}$ there exists a $\lambda>\lambda_{1}$ such that

$$
\alpha / \gamma<B
$$

We may assume that $-B$ is a continuity point of $G(x)$. Now choose $\lambda_{1}$ so that for all $\lambda>\lambda_{1}$,

$$
P\left[\frac{N-\alpha}{\gamma} \leqq-B\right]>G(-B)-\frac{G(-B)}{2}=\frac{G(-B)}{2}>0 ;
$$

then for some $\lambda>\lambda_{1}$ we have both (33) and (34), whence 


$$
0=P[N<0]=P\left[\frac{N-\alpha}{\gamma}<\frac{-\alpha}{\gamma}\right] \geqq P\left[\frac{N-\alpha}{\gamma} \leqq-B\right]>0,
$$

a contradiction. It follows that $\gamma=o(\alpha)$ and hence $\gamma=o\left(\sigma^{2}\right)$.

We shall now show that $\alpha \rightarrow \infty$. If not, then since $\gamma=o(\alpha)$, it follows that $\gamma \rightarrow 0$, which we shall show to be impossible.

From Tchebychef's inequality, $\gamma \rightarrow 0$ implies that

$$
P[|N-\alpha|<1 / 2] \rightarrow 1 \text {. }
$$

But there is at most one integer $k$ satisfying $|k-\alpha|<1 / 2$; denoting this integer by $k_{\lambda}$ we have

$$
P\left[N=k_{\lambda}\right] \rightarrow 1 .
$$

Define

$$
L=\liminf _{\lambda \rightarrow \infty}\left\{\frac{k_{\lambda}-\alpha}{\gamma}\right\} .
$$

Either $L>-\infty$ or $L=-\infty$. If the former, let $x<L$ be a continuity point of $G(x)$. Then

$$
G(x)=\lim _{\lambda \rightarrow \infty} P\left[\frac{N-\alpha}{\gamma} \leqq x\right] .
$$

But for sufficiently large $\lambda$,

$$
\frac{k_{\lambda}-\alpha}{\gamma}>x
$$

whence

$$
P\left[\frac{N-\alpha}{\gamma} \leqq x\right]<1-P\left[N=k_{\lambda}\right] .
$$

It follows that $G(x)=0$, a contradiction. On the other hand suppose $L=-\infty$. Then for any $x$ and sufficiently large $\lambda$,

$$
\frac{k_{\lambda}-\alpha}{\gamma}<x
$$

whence

$$
P\left[\frac{N-\alpha}{\gamma} \leqq x\right] \geqq P\left[N=k_{\lambda}\right] .
$$

It follows that $G(x)=1$. Since $x$ was arbitrary, $G(x)$ is not a d. f. 
Thus $\alpha \rightarrow \infty$ and hence $\sigma^{2} \rightarrow \infty$. This completes the proof.

It follows that in Corollary 3 we may drop the assumption (10) provided $G(x)>0$. Moreover, Corollary 2 may now be given its final form.

COROLlaRY 4. If $N$ is asymptotically normal $(\alpha, \gamma)$ then $Y$ is asymptotically normal $(\alpha a, \sigma)$.

We shall conclude this section with a theorem concerning the "singular" case in which $\alpha$ and $\gamma$ are of the same order as $\lambda \rightarrow \infty$, and $a=0$, so that (10) does not hold.

TheOREM 2. Let $a=0$. If as $\lambda \rightarrow \infty$

$$
\alpha \rightarrow \infty, \quad \gamma / \alpha \rightarrow r \quad(0<r<\infty),
$$

and if $M$ has a limiting d. $f . G(x)$ (necessarily such that $G(x)=0$ for some $x)$, then

$$
\lim _{\lambda \rightarrow \infty} \phi(t)=\int_{0}^{\infty} e^{-t^{2} y / 2} d G_{1}(y)=g_{1}\left(\frac{i t^{2}}{2}\right),
$$

where

$$
G_{1}(x)=G\left(\frac{x-1}{r}\right), \quad g_{1}(t)=\int_{0}^{\infty} e^{i t x} d G_{1}(x) .
$$

Thus the limiting d. f. of $Z$ is

$$
H(x)=\int_{0}^{\infty} H_{0}\left(\frac{x}{y^{1 / 2}}\right) d G_{1}(y) .
$$

Proof. We have for $a=0$,

$$
\phi(t)=\sum_{0}^{\infty} \omega_{k} \cdot f^{k}\left(\frac{t}{\left(\alpha c^{2}\right)^{1 / 2}}\right)=\sum_{0}^{\infty} \omega_{k}\left\{f^{\gamma}\left(\frac{t}{\left(\alpha c^{2}\right)^{1 / 2}}\right)\right\}^{k / 2} .
$$

Now as $\lambda \rightarrow \infty$,

$$
f^{\gamma}\left(\frac{t}{\left(\alpha c^{2}\right)^{1 / 2}}\right)=\left\{f^{\alpha}\left(\frac{t}{\left(\alpha c^{2}\right)^{1 / 2}}\right)\right\}^{\gamma / \alpha} \rightarrow e^{-r t^{2} / 2}
$$

Set

$$
\zeta=\frac{-i \log f^{r}\left(t /\left(\alpha c^{2}\right)^{1 / 2}\right)}{r}
$$

then as $\lambda \rightarrow \infty$, 


$$
\zeta \rightarrow i t^{2} / 2
$$

and

$$
\phi(t)=\sum_{0}^{\infty} \omega_{k} \cdot e^{i r \xi k / \gamma}
$$

Now let

$$
M_{1}=r N / \gamma
$$

then for any $x$ such that $(x-1) / r$ is a continuity point of $G(x)$,

$$
\begin{aligned}
P\left[M_{1} \leqq x\right]=P\left[\frac{r N}{\gamma} \leqq x\right] & =P\left[\frac{N-\alpha}{\gamma} \leqq \frac{x}{r}-\frac{\alpha}{\gamma}\right] \\
& \rightarrow G\left(\frac{x-1}{r}\right)=G_{1}(x),
\end{aligned}
$$

where $G_{1}(x)$ is defined by (37). It follows that

$$
E\left(e^{i z M 1}\right)=\sum_{0}^{\infty} \omega_{k} \cdot e^{i z r k / \gamma} \rightarrow g_{1}(z)=\int_{0}^{\infty} e^{i z y} d G_{1}(y)
$$

uniformly for every $z$ in some neighborhood of $z=i t^{2} / 2$. Hence from (39) and (40),

$$
\lim _{\lambda \rightarrow \infty} \phi(t)=g_{1}\left(\frac{i t^{2}}{2}\right)=\int_{0}^{\infty} e^{-t^{2} y / 2} d G_{1}(y) .
$$

Since

$$
\begin{aligned}
\int_{0}^{\infty} e^{-t^{2} y / 2} d G_{1}(y) & =\int_{0}^{\infty} \int_{-\infty}^{\infty} e^{i t x} d_{x} H_{0}\left(\frac{x}{y^{1 / 2}}\right) d G_{1}(y) \\
& =\int_{-\infty}^{\infty} e^{i t x} d x\left\{\int_{0}^{\infty} H_{0}\left(\frac{x}{y^{1 / 2}}\right) d G_{1}(y)\right\},
\end{aligned}
$$

it follows that the limiting d. f. of $Z$ is given by (38). This completes the proof of Theorem 2.

From the relation $M_{1}=r M+(r \alpha) / \gamma$ it follows that $g_{1}(t)=e^{i t} \cdot g(r t)$, where $g(t)$ is defined by (29). Hence (36) may be written in the equivalent form

$$
\lim _{\lambda \rightarrow \infty} \phi(t)=e^{-t^{2} / 2} \cdot g\left(\frac{i r t^{2}}{2}\right) .
$$

3. Some examples. (i) Let $N$ have a Poisson distribution with 
parameter $\lambda$, so that

$$
\omega_{k}=e^{-\lambda} \cdot\left(\lambda^{k} / k !\right) \quad(k=0,1, \cdots)
$$

then

$$
\alpha=\gamma^{2}=\lambda, \quad \sigma^{2}=\lambda b^{2} .
$$

From Corollary 4 it follows that $Y$ is asymptotically normal $\left(\lambda a, b \lambda^{1 / 2}\right)$. Note that (10) holds but (24) does not.

(ii) Let $N$ have a binomial distribution with parameters $\lambda, p$, where $\lambda$ is an arbitrary positive integer and $p$ and $q=1-p$ are constants, so that

$$
\omega_{k}=\frac{\lambda !}{k !(\lambda-k) !} p^{k} q^{\lambda-k} \quad(k=0,1, \cdots, \lambda)
$$

then

$$
\alpha=\lambda p, \quad \gamma^{2}=\lambda p q, \quad \sigma^{2}=\lambda p\left(c^{2}+q a^{2}\right) .
$$

Again it follows from Corollary 4 that $Y$ is asymptotically normal $\left(\lambda p a,\left(\lambda p\left(c^{2}+q a^{2}\right)\right)^{1 / 2}\right)$.

(iii) For any integer $\lambda$ suppose that $N$ can assume the two values $\lambda, 2 \lambda$, with probability $1 / 2$ in each case. Then

$$
\alpha=\frac{3 \lambda}{2}, \quad \gamma^{2}=\frac{\lambda^{2}}{4}, \quad \sigma^{2}=\frac{3 \lambda}{2} c^{2}+\frac{\lambda^{2}}{4} a^{2} .
$$

First suppose $a \neq 0$. Then as $\lambda \rightarrow \infty$ (10) holds, and the quantity $s$ of Corollary 3 is 0 . Moreover, $\theta(t)=\cos t$, so that $M$ has the non-normal limiting distribution for which $P[M=-1]=P[M=1]=1 / 2$. It follows from Corollary 3 that $Z$ has the same limiting distribution.

The case is quite different when $a=0$, for then $\gamma \neq o\left(\sigma^{2}\right)$, and Theorem 2 applies. We have

$$
r=\lim _{\lambda \rightarrow \infty} \frac{\gamma}{\alpha}=\frac{1}{3}, \quad g_{1}(t)=\frac{1}{2}\left\{e^{2 i t / 3}+e^{4 i t / 3}\right\},
$$

so that

$$
\lim _{\lambda \rightarrow \infty} \phi(t)=\frac{1}{2}\left\{e^{-t^{2} / 3}+e^{-2 t^{2} / 3}\right\} .
$$

Thus the limiting d. f. of $Z$ is a mixture of two normal d. f.'s with means 0 and variances $2 / 3$ and $4 / 3$.

University of North Carolina 